\title{
Cross-sectional and longitudinal assessment of arterial stiffening with age in European and Chinese populations
}

\section{Wiktoria Wojciechowska ${ }^{1}$, Yan Li ${ }^{2}$, Katarzyna Stolarz-Skrzypek ${ }^{1}$, Kalina Kawecka-Jaszcz', Jan A. Staessen ${ }^{3,4}$ * Ji-Guang Wang ${ }^{2}$ and on behalf of the European Project on Genes in Hypertension and the JingNing Study Investigators}

\author{
The First Department of Cardiology and Hypertension, Jagiellonian University Medical College, Kraków, Poland \\ ${ }^{2}$ Center for Epidemiologic Studies and Clinical Trials and Center for Vascular Evaluations, Shanghai Key Laboratory of Vascular Biology, Shanghai Institute of \\ Hypertension, Ruijin Hospital, Shanghai Jiaotong University School of Medicine, Shanghai, China \\ ${ }^{3}$ Division of Hypertension and Cardiovascular Rehabilitation, Department of Cardiovascular Diseases, Studies Coordinating Centre, University of Leuven, Leuven, \\ Belgium \\ ${ }^{4}$ Department of Epidemiology, Maastricht University, Maastricht, Netherlands
}

\section{Edited by:}

Steffen-Sebastian Bolz, University of

Toronto, Canada

Reviewed by:

Alun Hughes, Imperial College London, UK

Giovanni E. Mann, King's College

London, UK

*Correspondence:

Jan A. Staessen, Laboratory of Hypertension, Studies Coordinating

Centre, Campus Sint Rafaël,

Kapucijnenvoer 35, Block D, Box

7001, BE-3000 Leuven, Belgium.

e-mail: jan.staessen@

med.kuleuven.be;

jan.staessen@uzleuven.be;

ja.staessen@epid.unimaas.nl
As arteries become stiffer with aging, reflected waves move faster and augment late systolic pressure. Few studies have described the age-related changes in both peripheral and central systolic blood pressures in populations. We investigated the age dependency of peripheral (pSBP) and central (cSBP) systolic pressure and pressure amplification (i.e., difference between peripheral and central SBP) in randomly selected participants from European and Chinese populations. Data were collected in 1420 Europeans (mean age, 41.7 years) and 2044 (mean age, 45.1 years) Chinese. In cross-sectional analyses of the population samples cSBP consistently increased more with age than PSBP with the agerelated increases being greater in women than men. Repeat assessment of pSBP and cSBP in 398 Europeans and 699 Chinese at a median interval approximately 4 years of follow-up confirmed that also within subjects CSBP rose steeper with aging than pSBP. In conclusion, with aging, pSBP approximates to cSBP. This might explain why in older subjects pSBP becomes the main predictor of cardiovascular complications.

Keywords: aging, central blood pressure, peripheral blood pressure, cardiovascular disease, risk factors, epidemiology

\section{INTRODUCTION}

Systolic blood pressure substantially rises with aging across the full human life span. However, because arteries stiffen with higher age and because of differences in wave travel distance and wave reflections, the age-related increase in systolic blood pressure does not occur uniformly across the whole arterial tree (O'Rourke and Kelly, 1993). Indeed, during systole, the heart generates a forward running pressure wave, which is reflected at various sites in the peripheral arterial system (O'Rourke and Kelly, 1993). As the arteries become stiffer with advancing age, the reflected waves may cause an augmentation of late systolic pressure. In addition geometric changes of the aorta with aging may also contribute to age-related increase in systolic blood pressure (Redheuil et al., 2011). Additionally aortic reservoir function and other elastic changes markedly with aging, which accounts for the age-related changes in the aortic pressure waveform (Davies et al., 2010). Thus augmentation of systolic blood pressure in the central arteries increases with advancing age. Because of this phenomenon, there might be differences in the age dependency of peripheral systolic blood pressure, as measured at the brachial artery, and central systolic blood pressure, as estimated non-invasively by pulse wave analysis.

Systolic augmentation is now a generally accepted physiological concept. However, the initial evidence supporting the principle of the age dependency of the amplification of the central blood pressure came from animal experiments (O'Rourke, 1994) and invasive studies in selected subjects (Murgo et al., 1980; O’Rourke, 1994). Among the published population studies on the age dependency of arterial stiffness (Avolio et al., 1983, 1985; Wilkinson et al., 2001; Mitchell et al., 2004; McEniery et al., 2005; Li et al., 2008), most focused on pulse wave velocity (Avolio et al., 1983, 1985; Mitchell et al., 2004; McEniery et al., 2005), the ratio of pulse pressure in peripheral vs. central arteries (Wilkinson et al., 2001), or the augmentation index (McEniery et al., 2005; Li et al., 2008) in selected healthy subjects (Wilkinson et al., 2001; Mitchell et al., 2004; McEniery et al., 2005; Li et al., 2008) and patients at increased cardiovascular risk (McEniery et al., 2008). The predictive value of central pressure (both measured invasively and non-invasively) was proved in several studies (Roman et al., 2007; Jankowski et al., 2008). We previously reported reference values for the central and peripheral pulse pressures and augmentation indexes by age in healthy European (Wojciechowska et al., 2006) and Chinese (Li et al., 2008) reference populations. In the present review, we focused on the changes with age in central and peripheral systolic blood pressures, based on data collected from randomly recruited European (Wojciechowska et al., 2012) and Chinese (Li et al., 2012) subjects. These age-related changes were assessed cross-sectionally and in a subsample also longitudinally. Only participants without 
antihypertensive drug treatment were included in all analyses to avoid confounding by blood pressure lowering medications.

\section{CROSS-SECTIONAL STUDIES}

Peripheral and central blood pressures were assessed among 1420 participants [731 women (51.5\%) and 278 (19.6\%) hypertensive patients] in Europe and 2044 [1066 women (52.2\%) and 556 $(27.2 \%)$ hypertensive patients] in China (Wojciechowska et al., 2012; Li et al., 2012).

In cross-sectional analyses of both populations, the peripheral and central systolic blood pressures increased with age ( $p$ for trend $\leq$ 0.01; Figure 1 for Europe; Wojciechowska et al., 2012). In single regression analysis, the cross-sectionally assessed age-related increase in central systolic blood pressure was larger than that in peripheral systolic pressure both in women and in men. Additionally, in a subanalysis of the Chinese sample, systolic blood increased more with age in the central than peripheral arteries in women below age 50 ( 1.21 vs. $1.01 \mathrm{~mm} \mathrm{Hg}$ per year; $p<0.001)$ and in men below age 60 ( 0.73 vs. $0.48 \mathrm{~mm} \mathrm{Hg}$ per year; $p<0.001)$, whereas in older women $(0.64$ vs. $0.58 \mathrm{mmHg}$ per year; $p=0.27)$ and older men ( 0.45 vs. $0.44 \mathrm{~mm} \mathrm{Hg}$ per year; $p=0.79$ ), the slopes of central and peripheral systolic blood pressures on age were similar (Li et al., 2012).

Our cross-sectional observations are in agreement with known physiologic concepts and also in line with several previously published cross-sectional population studies (Wilkinson et al., 2001; McEniery et al., 2005; Mitchell et al., 2010), including the AngloCardiff Collaborative Trial (ACCT; McEniery et al., 2005). McEniery et al. (2005) studied 4001 healthy, normotensive individuals, aged 18-90 years. In both women and men, central systolic pressure increased more with age than did peripheral systolic blood pressure $(p<0.001)$. As in our current cross-sectional analyses, the increase in central systolic pressure was more prominent in women than men $(p=0.01)$. These consistent results were obtained based on White European populations with a western life style, high prevalence of obesity, and relatively high cholesterol levels. We cannot simply extrapolate them to other ethnicities or populations with different lifestyles. However, our findings were similar in lean Asian people, who generally have lower serum cholesterol levels.

Stiffening of the large arteries underlies the age-related increase in systolic blood pressure (Staessen et al., 1990). The loss of arterial elasticity over a person's life time is partly due to cyclic stress on the arterial wall with each heart beat (O'Rourke and Hashimoto, 2007). Over time, this causes fracture of elastin fibers, so that stress is transferred to the more rigid collagenous components of the arterial wall. At a young age, the aorta and proximal arteries dilate by approximately $10 \%$ with each heart beat, whereas the more distal muscular arteries dilate by only $2-3 \%$ with each heart beat (Boutouyrie et al., 1992). Atherosclerosis and inflammation thicken the arterial wall and contribute to arterial stiffening over and beyond the mechanical stress. To differentiate natural degeneration of the arterial wall from aging from disease, Avolio et al. (1983) highlighted the interest of studies of arterial properties in population studies with low cholesterol and low prevalence of atherosclerosis, such as Chinese. Avolio et al. (1985) contrasted the Chinese living in areas with low and high prevalence of hypertension, Guangzhou (4.9\%) and Beijing (15.6\%), respectively. In Guangzhou subjects, pulse wave velocity was consistently lower in the aorta, arm, and leg, and increased to a lesser degree with age, compared with Beijing subjects (Avolio et al., 1985). The contemporary cholesterol levels were $4.34 \mathrm{mmoll}^{-1}$ in Guangzhou subjects and $4.49 \mathrm{mmoll}^{-1}$ in Beijing subjects. In our Chinese population, cholesterol levels were of similar magnitude $\left(4.73 \mathrm{mmoll}^{-1}\right)$, but the prevalence of hypertension was much higher (27.2\%). Our current observations strengthen Avolio's hypothesis (Avolio et al., 1983, 1985) that slowly progressing degeneration of the arterial wall through cyclic stress is the main cause of the age-related increase in systolic blood pressure.

The slopes of peripheral and central systolic blood pressures on age were consistently steeper $(p<0.001)$ in women than in men in both studied populations (Li et al., 2012; Wojciechowska et al., 2012). In the young and middle-aged subjects, the age-related increase in systolic blood pressure ran a steeper course in women than men. At all ages, women have a higher heart rate than men (Smulyan et al., 2001). The smaller height of women may be a cardiovascular risk factor, because of the early return of reflected waves to the central aorta in systole. The shorter stature of women also implies reduced length of the arterial tree, a factor believed to be responsible for the faster heart rate, a shorter diastolic period, a shorter diastolic time constant, and at the same peripheral resistance, lower arterial compliance (Smulyan et al., 2001). In older women, menopause might contribute to the continuing rise in systolic blood pressure, although it is difficult to differentiate the effects of aging from those of estrogen deprivation (Casiglia et al., 2008).

\section{LONGITUDINAL ASSESSMENT OF AGE-DEPENDENT CHANGES IN ARTERIAL TREE}

In European study 208 women (24.4\%) and 190 men (27.6\%), underwent a repeat arterial examination at a median interval of 4.79 years ( 5 th- 95 th percentile interval, 3.96-5.98 years (Wojciechowska et al., 2012). In the Chinese study 369 women (34.6\%) and 330 men $(33.7 \%)$ underwent a repeat assessment of peripheral and central systolic blood pressure at a median interval of 3.60 years (5th-95th percentile interval, 3.56-3.96 years; Li et al., 2012). In the longitudinal analyses, all changes from baseline to follow-up were significant $(p \leq 0.039)$. In the Caucasian populations, the annual increases in the peripheral and central systolic blood pressures averaged 0.91 and $1.06 \mathrm{mmHg}$ in women, and 1.24 and $1.47 \mathrm{mmHg}$ in men. The $p$-values for the sex differences were 0.12 and 0.08 , respectively (Wojciechowska et al., 2012). In Chinese population peripheral systolic pressure increased more $(p \leq 0.025)$ than the central systolic pressure both in women ( $2.35 \mathrm{vs.} 2.12 \mathrm{~mm}$ $\mathrm{Hg}$ ) and in men ( 1.37 vs. $1.16 \mathrm{~mm} \mathrm{Hg}$ ). On a relative scale, the percentage increases in peripheral and central systolic blood pressure from baseline to follow-up were similar in women (2.14 vs. $2.16 \%$ per year; $p=0.76)$, as well as in men $(1.33$ vs. $1.34 \%$ per year; $p=0.96$;). In sensitivity analyses stratified by quartiles of the age distribution, the increase in peripheral systolic blood pressure was larger than that in central systolic blood pressure $(p \leq 0.02)$ above median age in women and above the 75th percentile of age in men, whereas in all other sex-age subgroups, the increases of peripheral and central systolic pressures were similar $(p \geq 0.08$; Figure 2 ; Li et al., 2012). 

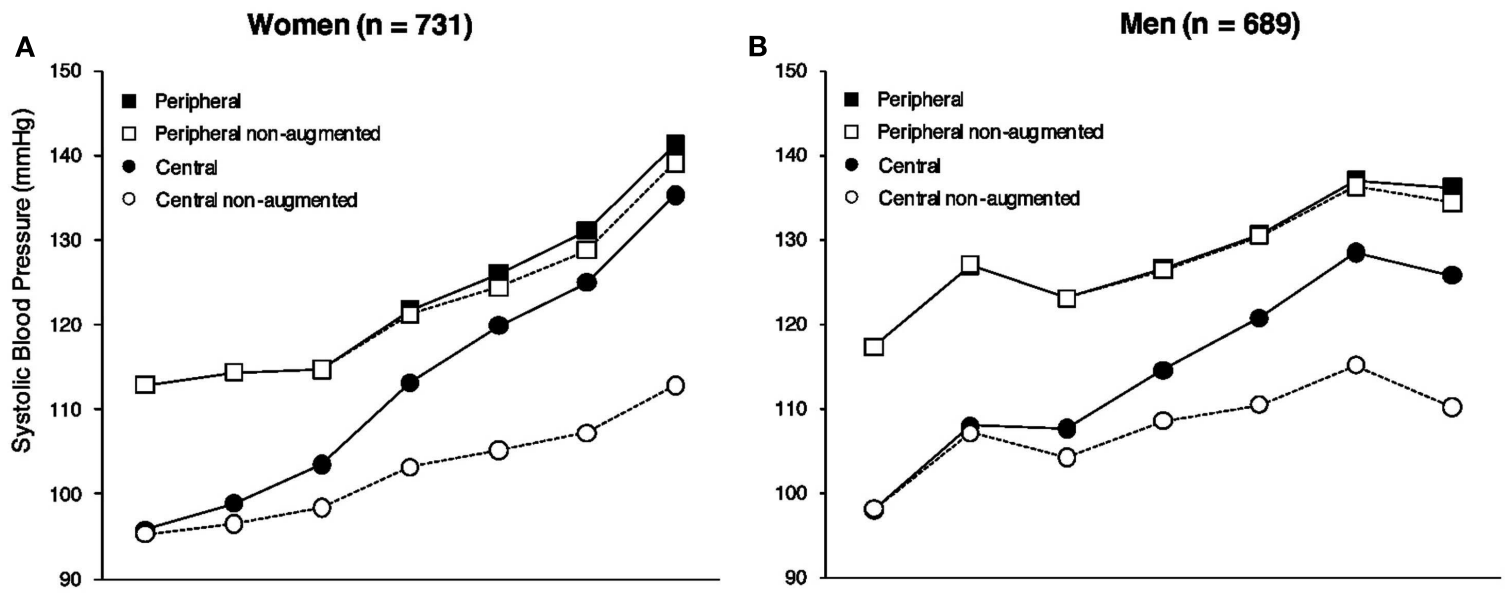

C

D
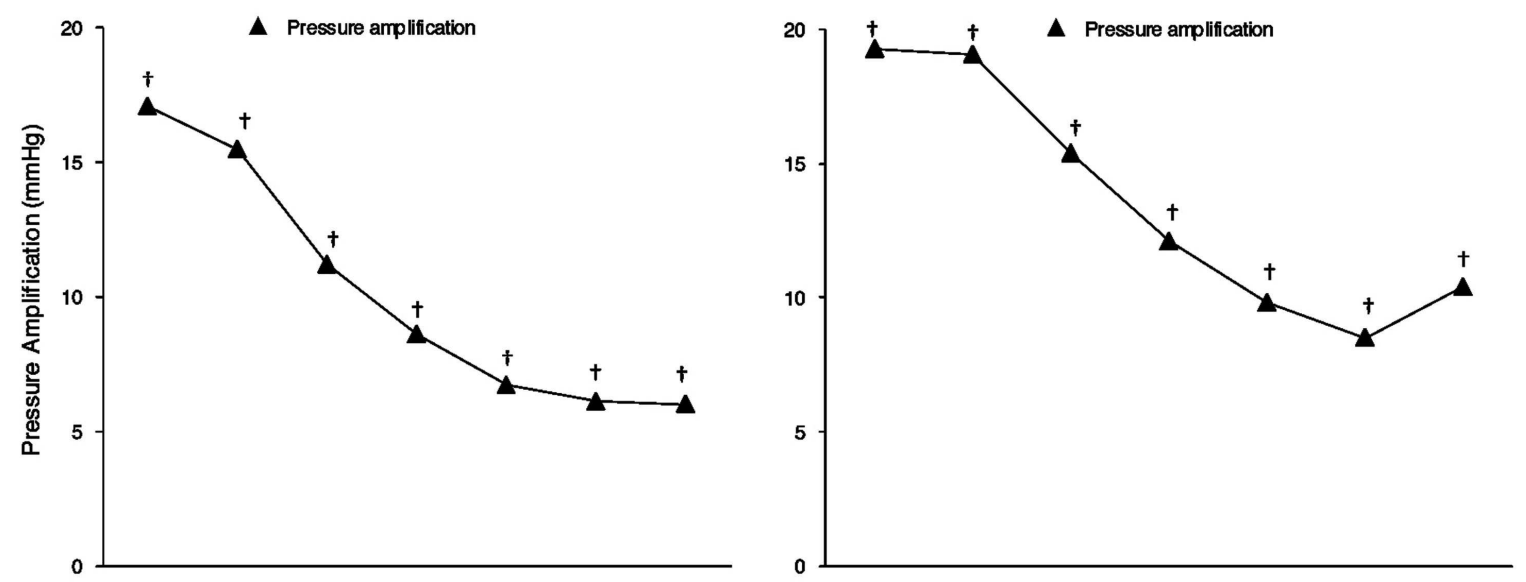

$\mathbf{E}$

$\mathbf{F}$
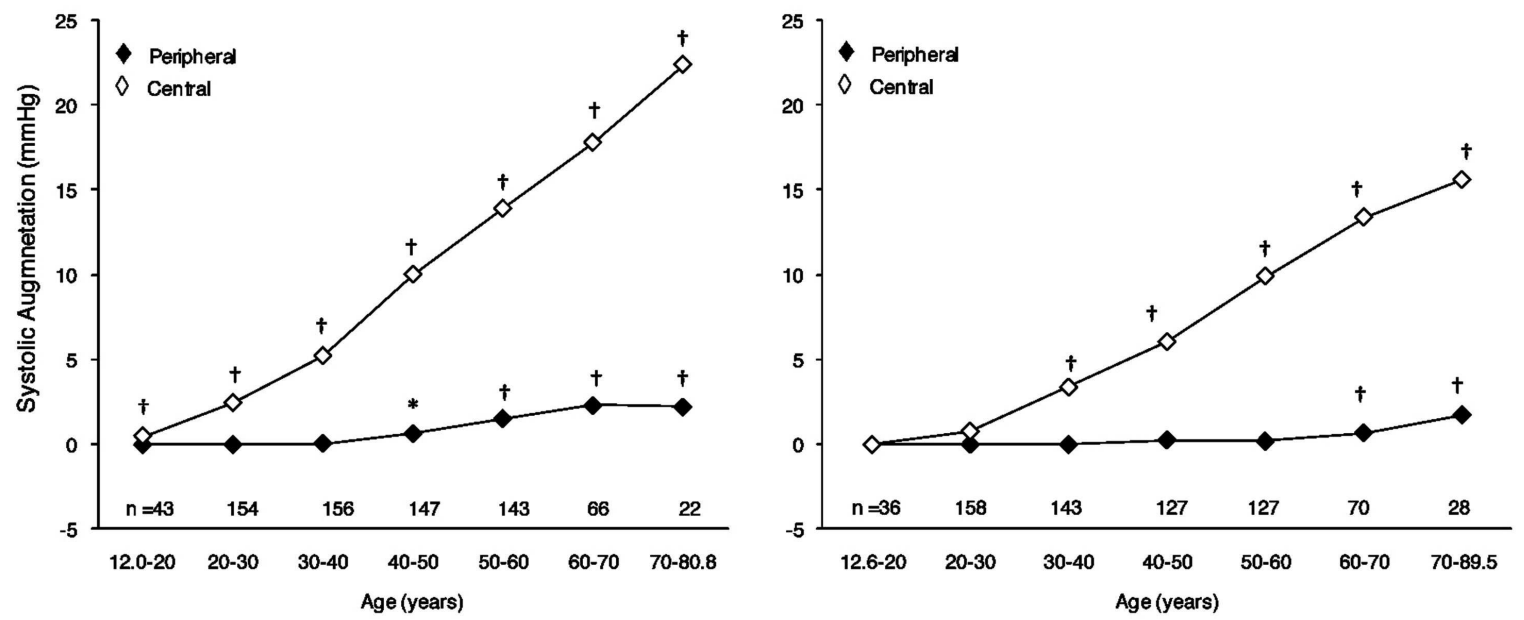

FIGURE 1 | European population. Association with age of peripheral and central systolic blood pressures $(\mathbf{A}, \mathbf{B})$, peripheral, and central non-augmented systolic blood pressures (A,B), pressure amplification (C,D), and peripheral and central systolic augmentation $\mathbf{( E , F ) ~ i n ~ w o m e n ~}(\mathbf{A}, \mathbf{C}, \mathbf{E})$ and men $(\mathbf{B}, \mathbf{D}, \mathbf{F})$. Peripheral systolic blood pressure was the average of three blood pressure readings at the brachial artery. Central systolic blood pressure was the maximum pressure of the central waveform. Systolic augmentation was

obtained by subtracting the first systolic peak from systolic blood pressure. Pressure amplification is peripheral minus central systolic blood pressure. Plotted values are means for each age group. Numbers indicate the subjects contributing to the group means. All $p$-values for trend with age were statistically significant $(p<0.0001)$. Significance of the difference with zero: ${ }^{*} p<0.05$ and ${ }^{\dagger} p<0.001$. Reproduced with permission from Blood Pressure Journal. 


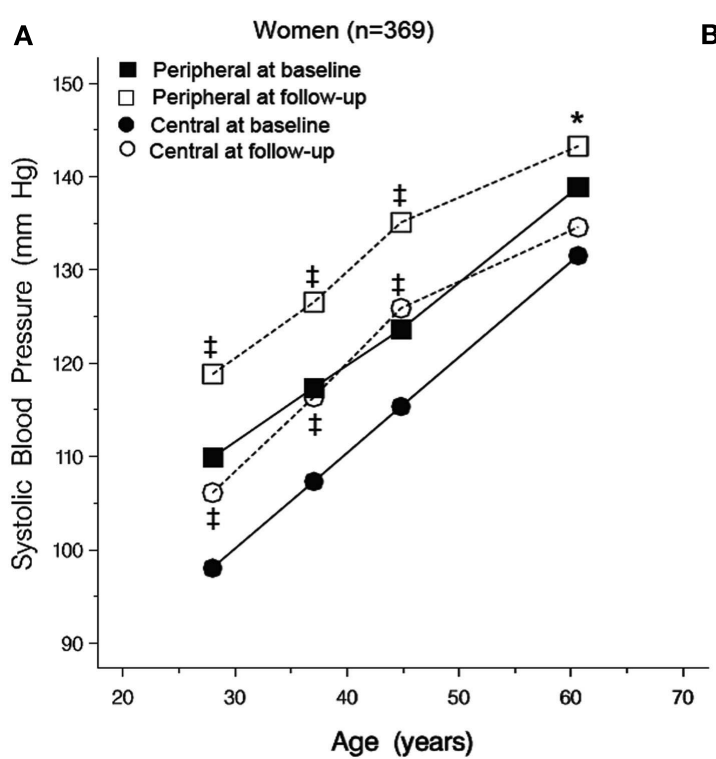

FIGURE 2 | Chinese population. Peripheral and central SBPS at baseline and follow-up by quartiles of the age distribution in 369 women (A) and 330 men (B). All $p$-values for trend with age were statistically significant $(p<0.0001)$.

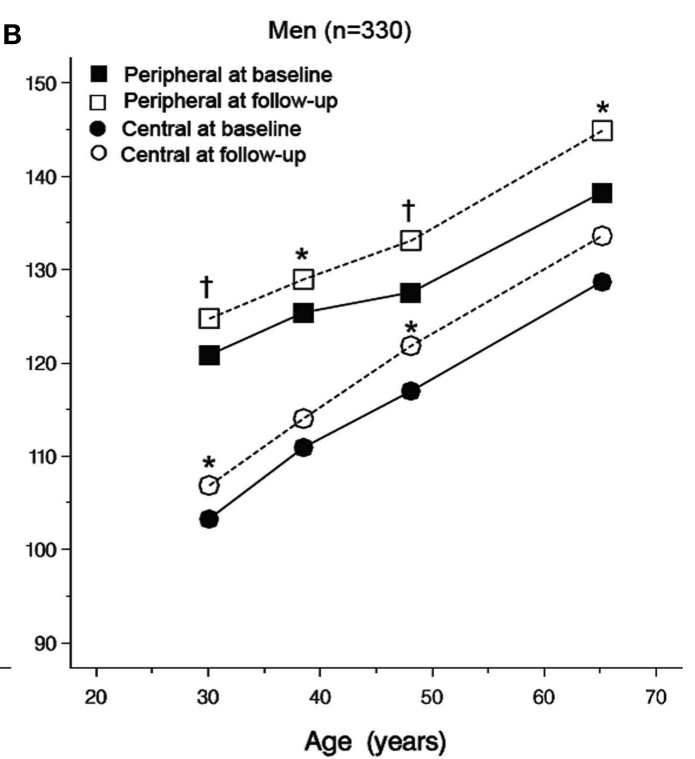

Significance of the difference between baseline and follow-up: ${ }^{*} p<0.05$, ${ }^{\ddagger} p<0.01$, and ${ }^{\dagger} p<0.001$. Reproduced with permission from Hypertension Research Journal.
In an early Framingham report, Kannel and Gordan (1978) noticed that the age-related increase in systolic blood pressure was steeper on cross-sectional than longitudinal assessment in women, whereas the opposite was the case in men. The reasons for the difference in BP trends obtained cross-sectionally and longitudinally in the same Framingham cohort were not clear. One possible explanation is that subjects at the higher end of the distribution of systolic blood pressure are more likely to experience cardiovascular complications or die, and therefore to disappear from follow-up. The unmeasured attrition in our populations available for cross-sectional analysis might have contributed to the larger estimates of the age-related increase in systolic blood pressure in the longitudinal analyses.

\section{REFERENCES}

Avolio, A. P., Chen, S. G., Wang, R. P., Zhang, C. L., Li, M. F., and O'Rourke, M. F. (1983). Effects of aging on changing arterial compliance and left ventricular load in a northern Chinese urban community. Circulation 68, 50-58.

Avolio, A. P., Deng, F. A., Li, W. Q., Luo, Y. F., Huang, Z. D., Xing, L. F., and O'Rourke, M. F. (1985). Effects of aging on arterial distensibility in populations with high and low prevalence of hypertension: comparison between urban and rural communities in China. Circulation 71, 202-210.

Boutouyrie, P. H., Laurent, S., Benetos, A., Girerd, X. J., Hoeks, A. P. G., and Safar, M. E. (1992). Opposing effects of ageing on distal and proximal large arteries in hypertensives. J. Hypertens. 10, S87-S91.

Casiglia, E., Tikhonoff, V., Caffi, S., Bascelli, A., Schiavon, L., Guidotti, F., Saugo, M., Giacomazzo, M., Martini, B., Mazza, A., D'Este, D., and Pessina, A. C. (2008). Menopause does not affect blood pressure and risk profile, and menopausal women do not become similar to men. J. Hypertens. 26, 1983-1992.

Davies, J. E., Baksi, J., Francis, D. P., Hadjiloizou, N., Whinnett, Z. I., Manisty, C. H., Aguado-Sierra, J., Foale, R. A., Malik, I. S., Tyberg, J. V., Parker, K. H., Mayet, J., and Hughes, A. D. (2010). The arterial reservoir pressure increases with aging and is the major determinant of the aortic augmentation index. Am.

\section{CONCLUSION}

The effects of aging on arterial function have often been underestimated, because of the sole reliance on the brachial cuff systolic pressure. With aging, peripheral systolic blood pressure approximates to the central systolic blood pressure. These findings support the point of view that age-related stiffness of arteries represents a vicious circle, in which increasing systolic blood pressure is, at the same time, the cause and the consequence of a self-sustaining process that leads to major cardiovascular complications. Breaking the vicious circle is the key to slowing the age-related rise in systolic blood pressure and preventing the associated cardiovascular complications.

J. Physiol. Heart Circ. Physiol. 298, H580-H586.

Jankowski, P., Kawecka-Jaszcz, K., Czarnecka, D., Brzozowska-Kiszka, M., Styczkiewicz, K., Loster, M., KlochBadelek, M., Wiliński, J., Curylo, A. M., Dudek, D., and Aortic Blood Pressure and Survival Study Group. (2008). Pulsatile but not steady component of blood pressure predicts cardiovascular events in coronary patients. Hypertension 51, 848-855.

Kannel, W. B., and Gordan, T. (1978). Evaluation of cardiovascular risk in the elderly: the Framingham study. Bull. N. Y. Acad. Med. 54, 573-591.

Li, Y., Staessen, J. A., Li, L. H., Huang, Q. F., Lu, L., and Wang, J. G. (2008). Reference values for the arterial pulse wave in Chinese. Am. J. Hypertens. $21,668-673$.
Li, Y., Staessen, J. A., Sheng, C. S., Huang, Q. F., O'Rourke, M., and Wang, J. G. (2012). Age dependency of peripheral and central systolic blood pressures: cross-sectional and longitudinal observations in a Chinese population. Hypertens. Res. 35, 115-122.

McEniery, C. M., Yasmin, McDonnell, B., Munnery, M., Wallace, S. M., Rowe, C. V., Cockcroft, J. R., Wilkinson, I. B., and AngloCardiff Collaborative Trial Investigators. (2008). Central pressure: variability and impact of cardiovascular risk factors: the Anglo-Cardiff Collaborative Trial II. Hypertension 51, 1476-1482.

McEniery, C. M., Yasmin Hall, I. R., Qasem, A., Wilkinson, I. B., and Cockcroft, J. R., and ACCT Investigators. (2005). Normal vascular 
aging: differential effects on wave reflection and aortic pulse wave velocity. The Anglo-Cardiff Collaborative Trial (ACCT). J. Am. Coll. Cardiol. 46, 1753-1760.

Mitchell, G. F., Parise, H., Benjamin, E. J., Larson, M. G., Keyes, M. J., Vita, J. A., Vasan, R. S., and Levy, D. (2004). Changes in arterial stiffness and wave reflection with advancing age in healthy men and women. The Framingham Heart Study. Hypertension 43, 1239-1245.

Mitchell, G. F., Wang, N., Palmisano, J. N., Larson, M. G., Hamburg, N. M., Vita, J. A., Levy, D., Benjamin, E. J., and Vasan, R. S. (2010). Hemodynamic correlates of blood pressure across the adult age spectrum: noninvasive evaluation in the Framingham Heart Study. Circulation 122, 1379-1386.

Murgo, J. P., Westerhof, N., Giolma, J. P., and Altobelli, S. A. (1980). Aortic input impedance in normal man: relationship to pressure wave forms. Circulation 62, 105-116.

O'Rourke, M. (1994). Arterial haemodynamics and ventricular-vascular interaction in hypertension. Blood Press. 3, 33-37.

O'Rourke, M. F., and Hashimoto, J. (2007). Mechanical factors in arterial aging. A clinical perspective. J. Am. Coll. Cardiol. 50, 1-13.

O’Rourke, M. F., and Kelly, P. J. (1993). Wave reflections in the systemic circulation and its implications in ventricular function. J. Hypertens. 11, 327-337.

Redheuil, A., Yu, W. C., Mousseaux, E., Harouni, A. A., Kachenoura, N., Wu, C. O., Bluemke, D., and Lima, J. A. (2011). Age-related changes in aortic arch geometry: relationship with proximal aortic function and left ventricular mass and remodeling. J. Am. Coll. Cardiol. 58, 1262-1270.

Roman, M. J., Devereux, R. B., Kizer, J. R., Lee, E. T., Galloway, J. M., Ali, T., Umans, J. G., and Howard, B. V. (2007). Central pressure more strongly relates to vascular disease and outcome than does brachial pressure. The Strong Heart study. Hypertension 50, 197-203.

Smulyan, H., Asmar, R. G., Rudnicki, A., London, G. M., and Safar, M. E. (2001). Comparative effects of aging in men and women on the properties of the arterial tree. J. Am. Coll. Cardiol. 37, 1374-1380.

Staessen, J., Amery, A., and Fagard, R. (1990). Editorial review. Isolated systolic hypertension in the elderly. J. Hypertens. 8, 393-405.
Wilkinson, I. B., Franklin, S. S., Hall, I. R., Tyrrell, S., and Cockcroft, J. R. (2001). Pressure amplification explains why pulse pressure is unrelated to risk in young subjects. Hypertension 38, 1461-1466.

Wojciechowska, W., Staessen, J. A. Nawrot, T., Cwynar, M., Seidlerová, J., Stolarz, K., Gasowski, J., Tichá, M., Richart, T., Thijs, L., Grodzicki, T., Kawecka-Jaszcz, K., Filipovský, J., and European Project on Genes in Hypertension (EPOGH) Investigators. (2006). Reference values in White Europeans for the arterial pulse wave recorded by means of the ShygmoCor device. Hypertens. Res. 29, 475-483.

Wojciechowska, W., Stolarz-Skrzypek, K., Tikhonoff, V., Richart, T., Seidlerová, J., Cwynar, M., Thijs, L., Li, Y., Kuznetsova, T., Filipovský, J. Casiglia, E., Grodzicki, T., KaweckaJaszcz, K., O’Rourke, M., Staessen, J. A., and European Project on Genes in Hypertension (Epogh) Investigators. (2012). Age dependency of central and peripheral systolic blood pressures: cross-sectional and longitudinal observations in European populations. Blood Press. 21, 58-68.

Conflict of Interest Statement: The authors declare that the research was conducted in the absence of any commercial or financial relationships that could be construed as a potential conflict of interest.

Received: 20 February 2012; accepted: 28 May 2012; published online: 15 June 2012.

Citation: Wojciechowska W, Li Y, Stolarz-Skrzypek K, Kawecka-Jaszcz K, Staessen JA, Wang J-G and on behalf of the European Project on Genes in Hypertension and the JingNing Study Investigators (2012) Cross-sectional and longitudinal assessment of arterial stiffening with age in European and Chinese populations. Front. Physio. 3:209. doi: 10.3389/fphys.2012.00209

This article was submitted to Frontiers in Vascular Physiology, a specialty of Frontiers in Physiology.

Copyright (C) 2012 Wojciechowska, Li, Stolarz-Skrzypek, Kawecka-Jaszcz, Staessen, Wang and on behalf of the European Project on Genes in Hypertension and the JingNing Study Investigators. This is an open-access article distributed under the terms of the Creative Commons Attribution Non Commercial License, which permits non-commercial use, distribution, and reproduction in other forums, provided the original authors and source are credited. 